\title{
End-to-end cross-modality retrieval with CCA projections and pairwise ranking loss
}

\author{
Matthias Dorfer ${ }^{1}(\mathbb{D}) \cdot$ Jan Schlüter ${ }^{2} \cdot$ Andreu Vall $^{1} \cdot$ Filip Korzeniowski $^{1} \cdot$ Gerhard Widmer $^{1,2}$
}

Received: 8 November 2017 / Revised: 14 February 2018 / Accepted: 24 February 2018 / Published online: 7 March 2018

(c) The Author(s) 2018. This article is an open access publication

\begin{abstract}
Cross-modality retrieval encompasses retrieval tasks where the fetched items are of a different type than the search query, e.g., retrieving pictures relevant to a given text query. The state-of-the-art approach to cross-modality retrieval relies on learning a joint embedding space of the two modalities, where items from either modality are retrieved using nearest-neighbor search. In this work, we introduce a neural network layer based on canonical correlation analysis (CCA) that learns better embedding spaces by analytically computing projections that maximize correlation. In contrast to previous approaches, the CCA layer allows us to combine existing objectives for embedding space learning, such as pairwise ranking losses, with the optimal projections of CCA. We show the effectiveness of our approach for cross-modality retrieval on three different scenarios (text-to-image, audio-sheet-music and zero-shot retrieval), surpassing both Deep CCA and a multi-view network using freely learned projections optimized by a pairwise ranking loss, especially when little training data is available (the code for all three methods is released at: https://github.com/CPJKU/cca_layer).
\end{abstract}

Keywords Cross-modality retrieval $\cdot$ Canonical correlation analysis $\cdot$ Ranking loss $\cdot$ Neural network $\cdot$ Joint embedding space

\section{Introduction}

Cross-modality retrieval is the task of retrieving relevant items of a different modality than the search query (e.g., retrieving an image given a text query). One approach to tackle this problem is to define transformations which embed samples from different modalities into a common vector space. We can then project a query into this embedding space, and retrieve, using nearest-neighbor search, a corresponding candidate projected from another modality.

A particularly successful class of models uses parametric nonlinear transformations (e.g., neural networks) for the embedding projections, optimized via a retrieval-specific objective such as a pairwise ranking loss $[15,27]$. This loss

Electronic supplementary material The online version of this article (https://doi.org/10.1007/s13735-018-0151-5) contains supplementary material, which is available to authorized users.

Matthias Dorfer

matthias.dorfer@jku.at

1 Department of Computational Perception, Johannes Kepler University Linz, 4040 Linz, Austria

2 The Austrian Research Institute for Artificial Intelligence, 1010 Vienna, Austria aims at decreasing the distance (a differentiable function such as Euclidean or cosine distance) between matching items, while increasing it between mismatching ones. Specialized extensions of this loss achieved state-of-the-art results in various domains such as natural language processing [10], image captioning [12], and text-to-image retrieval [29].

In a different approach, Yan and Mikolajczyk [31] propose to learn a joint embedding of text and images using Deep canonical correlation analysis (DCCA) [2]. Instead of a pairwise ranking loss, DCCA directly optimizes the correlation of learned latent representations of the two views. Given the correlated embedding representations of the two views, it is possible to perform retrieval via cosine distance. The promising performance of their approach is also in line with the findings of Costa et al. [23] who state the following two hypotheses regarding the properties of efficient crossmodal retrieval spaces: first, the embedding spaces should account for low-level cross-modal correlations and second, they should enable semantic abstraction. In [31], both properties are met by a deep neural network-learning abstract representations - that is optimized with DCCA ensuring highly correlated latent representations.

In summary, the optimization of pairwise ranking losses yields embedding spaces that are useful for retrieval, and 


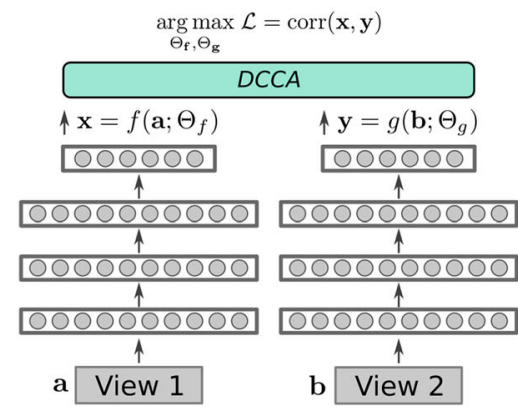

(a)

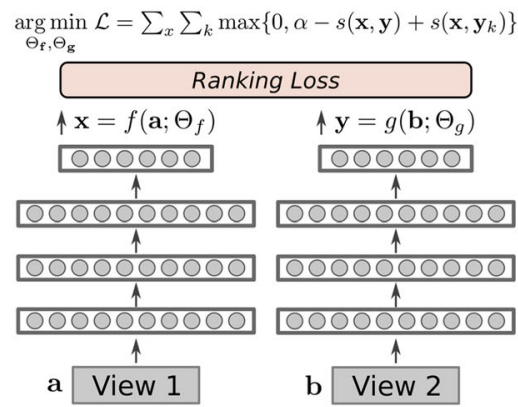

(b)

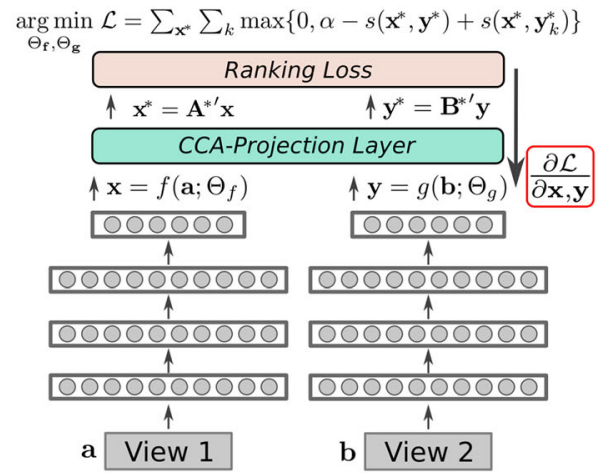

(c)
Fig. 1 Sketches of cross-modality retrieval networks. The proposed model in (c) unifies $(\mathbf{a}, \mathbf{b})$ and takes advantage of both componentwisecorrelated CCA projections and a pairwise ranking loss for crossmodality embedding space learning. We emphasize that our proposal in (c) requires to backpropagate the ranking loss $\mathcal{L}$ through the analytical computation of the optimally correlated CCA embedding projections $\mathbf{A}^{*}$

allows incorporating domain knowledge into the loss function. On the other hand, DCCA is designed to maximize correlation - which has already proven to be useful for crossmodality retrieval [31] - but does not allow to use loss formulations specialized for the task at hand.

In this paper, we propose a method to combine both approaches in a way that retains their advantages. We develop a Canonical Correlation Analysis Layer (CCAL) that can be inserted into a dual-view neural network to produce a maximally correlated embedding space for its latent representations. We can then apply task-specific loss functions, in particular the pairwise ranking loss, on the output of this layer. To train a network using the CCA layer, we describe how to backpropagate the gradient of this loss function to the dual-view neural network while relying on automatic differentiation tools such as Theano [28] or Tensorflow [1]. In our experiments, we show that our proposed method performs better than DCCA and models using pairwise ranking loss alone, especially when little training data is available.

Figure 1 compares our proposed approach to the alternatives discussed above. DCCA defines an objective optimizing a dual-view neural network such that its two views will be maximally correlated (Fig. 1a). Pairwise ranking losses are loss functions to optimize a dual-view neural network such that its two views are well-suited for nearest-neighbor retrieval in the embedding space (Fig. 1b). In our approach, we boost optimization of a pairwise ranking loss based on cosine distance by placing a special-purpose layer, the CCA projection layer, between a dual-view neural network and the optimization target (Fig. 1c). Our experiments in Sect. 5 will show the effectiveness of this proposal. and $\mathbf{B}^{*}$ (see Eq. 4). We thus need to compute their partial derivatives with respect to the network's hidden representations $\mathbf{x}$ and $\mathbf{y}$, i.e., $\frac{\partial \mathbf{A}^{*}}{\partial \mathbf{x}, \mathbf{y}}$ and $\frac{\partial \mathbf{B}^{*}}{\partial \mathbf{x}, \mathbf{y}}$ (addressed in Sect. 4). a DCCA network maximizes correlation via Trace Norm Objective $(T N O)$. b Freely learned embedding projections optimized with ranking loss (Learned $-\mathcal{L}_{\text {rank }}$ ). c Canonically correlated projection layer optimized with ranking loss $\left(C C A L-\mathcal{L}_{\text {rank }}\right)$

\section{Canonical correlation analysis}

In this section, we review the concepts of CCA, the basis for our methodology. Let $\mathbf{x} \in \mathbb{R}^{d_{x}}$ and $\mathbf{y} \in \mathbb{R}^{d_{y}}$ denote two random column vectors with covariances $\Sigma_{x x}$ and $\Sigma_{y y}$ and cross-covariance $\Sigma_{x y}$. The objective of CCA is to find two matrices $\mathbf{A}^{*} \in \mathbb{R}^{d_{x} \times k}$ and $\mathbf{B}^{*} \in \mathbb{R}^{d_{y} \times k}$ composed of $k$ paired column vectors $\mathbf{A}_{j}$ and $\mathbf{B}_{j}$ (with $k \leq d_{x}$ and $k \leq d_{y}$ ) that project $\mathbf{x}$ and $\mathbf{y}$ into a common space maximizing their componentwise correlation:

$$
\begin{aligned}
\left(\mathbf{A}^{*}, \mathbf{B}^{*}\right) & =\underset{\mathbf{A}, \mathbf{B}}{\arg \max } \sum_{j=1}^{k} \operatorname{corr}\left(\mathbf{A}_{j}^{\prime} \mathbf{x}, \mathbf{B}_{j}^{\prime} \mathbf{y}\right) \\
& =\underset{\mathbf{A}, \mathbf{B}}{\arg \max } \sum_{j=1}^{k} \frac{\mathbf{A}_{j}^{\prime} \Sigma_{x y} \mathbf{B}_{j}}{\sqrt{\mathbf{A}_{j}^{\prime} \Sigma_{x x} \mathbf{A}_{j} \mathbf{B}_{j}^{\prime} \Sigma_{y y} \mathbf{B}_{j}}} .
\end{aligned}
$$

Since the objective of CCA is invariant to scaling of the projection matrices, we constrain the projected dimensions to have unit variance. Furthermore, CCA seeks subsequently uncorrelated projection vectors, arriving at the equivalent formulation:

$$
\left(\mathbf{A}^{*}, \mathbf{B}^{*}\right)=\underset{\mathbf{A}^{\prime} \Sigma_{x x} \mathbf{A}=\mathbf{B}^{\prime} \Sigma_{y y} \mathbf{B}=\mathbf{I}_{k}}{\arg \max } \operatorname{tr}\left(\mathbf{A}^{\prime} \Sigma_{x y} \mathbf{B}\right) .
$$

Let $\mathbf{T}=\Sigma_{x x}^{-1 / 2} \Sigma_{x y} \Sigma_{y y}^{-1 / 2}$, and let $\mathbf{U} \operatorname{diag}(\mathbf{d}) \mathbf{V}^{\prime}$ be the Singular Value Decomposition (SVD) of T with ordered singular values $d_{i} \geq d_{i+1}$. As shown in [19], we obtain $\mathbf{A}^{*}$ and $\mathbf{B}^{*}$ from the top $k$ left- and right-singular vectors of $\mathbf{T}$ : 


$$
\mathbf{A}^{*}=\Sigma_{x x}^{-1 / 2} \mathbf{U}_{: k} \quad \mathbf{B}^{*}=\Sigma_{y y}^{-1 / 2} \mathbf{V}_{: k} .
$$

Moreover, the correlation in the projection space is the sum of the top $k$ singular values: ${ }^{1}$

$$
\operatorname{corr}\left(\mathbf{A}^{* \prime} \mathbf{x}, \mathbf{B}^{* \prime} \mathbf{y}\right)=\sum_{i \leq k} d_{i}
$$

In practice, the covariances and cross-covariance of $\mathbf{x}$ and $\mathbf{y}$ are usually not known, but estimated from a training set of $m$ paired vectors, expressed as matrices $\mathbf{X} \in \mathbb{R}^{d_{x} \times m}, \mathbf{Y} \in$ $\mathbb{R}^{d_{y} \times m}$ by:

$\hat{\Sigma}_{x x}=\frac{1}{m-1} \overline{\mathbf{X}} \overline{\mathbf{X}}^{\prime}+r \mathbf{I}$ and $\hat{\Sigma}_{x y}=\frac{1}{m-1} \overline{\mathbf{X}} \overline{\mathbf{Y}}^{\prime}$.

$\overline{\mathbf{X}}$ is the centered version of $\mathbf{X} . \hat{\Sigma}_{y y}$ is defined analogously to $\hat{\Sigma}_{x x}$. Additionally, we apply a regularization parameter $r \mathbf{I}$ to ensure that the covariance matrices are positive definite. Substituting these estimates for $\Sigma_{x x}, \Sigma_{x y}$ and $\Sigma_{y y}$, respectively, we can compute $\mathbf{A}^{*}$ and $\mathbf{B}^{*}$ using Eq. (4).

\section{Cross-modality retrieval baselines}

In this section, we review the two most related works forming the basis for our approach.

\subsection{Deep canonical correlation analysis}

Andrew et al. [2] propose an extension of CCA to learn parametric nonlinear transformations of two random vectors, such that their correlation is maximized. Let $\mathbf{a} \in \mathbb{R}^{d_{a}}$ and $\mathbf{b} \in \mathbb{R}^{d_{b}}$ denote two random vectors, and let $\mathbf{x}=f\left(\mathbf{a} ; \Theta_{f}\right)$ and $\mathbf{y}=g\left(\mathbf{b} ; \Theta_{g}\right)$ denote their nonlinear transformations, parameterized by $\Theta_{f}$ and $\Theta_{g}$. DCCA optimizes the parameters $\Theta_{f}$ and $\Theta_{g}$ to maximize the correlation of the topmost hidden representations $\mathbf{x}$ and $\mathbf{y}$. For $d_{x}=d_{y}=k$, this objective corresponds to Eq. 5, i.e., the sum of all singular values of $\mathbf{T}$, also called the trace norm:

$\operatorname{corr}\left(\mathbf{A}^{* \prime} f\left(\mathbf{a} ; \Theta_{f}\right), \mathbf{B}^{* \prime} g\left(\mathbf{b} ; \Theta_{g}\right)\right)=\|\mathbf{T}\|_{\mathrm{tr}}$.

Andrew et al. [2] show how to compute the gradient of this Trace Norm Objective (TNO) with respect to $\mathbf{x}$ and $\mathbf{y}$. Assuming $f$ and $g$ are differentiable with respect to $\Theta_{f}$ and $\Theta_{g}$ (as is the case for neural networks), this allows to optimize the nonlinear transformations via gradient-based methods.

Yan and Mikolajczyk [31] suggest the following procedure to utilize DCCA for cross-modality retrieval: first,

\footnotetext{
${ }^{1}$ We understand the correlation of two vectors to be defined as $\operatorname{corr}(\mathbf{x}, \mathbf{y})=\sum_{i} \sum_{j} \operatorname{corr}\left(x_{i}, y_{j}\right)$.
}

\begin{tabular}{|c|c|}
\hline 1. Maximize correlation by TNO & $\begin{array}{c}\text { Network } \\
\text { Optimization }\end{array}$ \\
\hline \begin{tabular}{c|c} 
2. Compute output of trained \\
networks and estimate
\end{tabular} & $\begin{array}{c}\text { Retrieval } \\
\text { CCA projection matrices }\end{array}$ \\
\hline Preparation \\
\hline 3. Project data to retrieval space & \\
\hline 4. Retrieval by cosine distance & Retrieval \\
\hline
\end{tabular}

Fig. 2 DCCA retrieval pipeline proposed in [31]. Note that all processing steps below the solid line are performed after network optimization is complete

neural networks $f$ and $g$ are trained using the TNO, with a and $\mathbf{b}$ representing different views of an entity (e.g. image and text); then, after the training is finished, the CCA projections are computed using Eq. (4), and all retrieval candidates are projected into the embedding space; finally, at test time, queries of either modality are projected into the embedding space, and the best-matching sample from the other modality is found through nearest-neighbor search using the cosine distance. Figure 2 provides a summary of the entire retrieval pipeline. In our experiments, we will refer to this approach as DCCA-2015.

DCCA is limited by design to use the objective function described in Eq. (7), and only seeks to maximize the correlation in the embedding space. During training, the CCA projection matrices are never computed, nor are the samples projected into the common retrieval space. All the retrieval steps - most importantly, the computation of CCA projections - are performed only once after the networks $f$ and $g$ have been optimized. This restricts potential applications, because we cannot use the projected data as an input to subsequent layers or task-specific objectives. We will show how our approach overcomes this limitation in Sect. 4.

\subsection{Pairwise ranking loss}

Kiros et al. [15] learn a multi-modal joint embedding space for images and text. They use the cosine of the angle between two corresponding vectors $\mathbf{x}$ and $\mathbf{y}$ as a scoring function, i.e., $s(\mathbf{x}, \mathbf{y})=\cos (\mathbf{x}, \mathbf{y})$. Then, they optimize a pairwise ranking loss

$$
\mathcal{L}_{\text {rank }}=\sum_{\mathbf{x}} \sum_{k} \max \left\{0, \alpha-s(\mathbf{x}, \mathbf{y})+s\left(\mathbf{x}, \mathbf{y}_{k}\right)\right\}
$$

where $\mathbf{x}$ is an embedded sample of the first modality, $\mathbf{y}$ is the matching embedded sample of the second modality, and $\mathbf{y}_{k}$ are the contrastive (mismatching) embedded samples of the second modality (in practice, all mismatching samples in the current mini-batch). The hyper-parameter $\alpha$ defines the margin of the loss function. This loss encourages an embedding 
space where the cosine distance between matching samples is lower than the cosine distance of mismatching samples.

In this setting, the networks $f$ and $g$ have to learn the embedding projections freely from randomly initialized weights. Since the projections are learned from scratch by optimizing a ranking loss, in our experiments, we denote this approach by Learned- $\mathcal{L}_{\text {rank }}$. Figure $1 \mathrm{~b}$ shows a sketch of this paradigm.

\section{Learning with canonically correlated embedding projections}

In the following, we explain how to bring both conceptsDCCA and Pairwise Ranking Losses-together to enhance cross-modality embedding space learning.

\subsection{Motivation}

We start by providing an intuition on why we expect this combination to be fruitful: DCCA-2015 maximizes the correlation between the latent representations of two different neural networks via the TNO derived from classic CCA. As correlation and cosine distance are related, we can also use such a network for cross-modality retrieval [31]. Kiros et al. [15], on the other hand, learn a cross-modality retrieval embedding by optimizing an objective customized for the task at hand. The motivation for our approach is that we want to benefit from both: a task-specific retrieval objective, and componentwise optimally correlated embedding projections.

To achieve this, we devise a CCA layer that analytically computes the CCA projections $\mathbf{A}^{*}$ and $\mathbf{B}^{*}$ during training, and projects incoming samples into the embedding space. The projected samples can then be used in subsequent layers, or for computing task-specific losses such as the pairwise ranking loss. Figure 1c illustrates the central idea of our combined approach. Compared to Fig. 1b, we insert an additional linear transformation. However, this transformation is not learned (otherwise it could be merged with the previous layer, which is not followed by a nonlinearity). Instead, it is computed to be the transformation that maximizes componentwise correlation between the two views. $\mathbf{A}^{*}$ and $\mathbf{B}^{*}$ in Fig. $1 \mathrm{c}$ are the very projections given by Eq. (4) in Sect. 2 .

In theory, optimizing a pairwise ranking loss alone could yield projections equivalent to the ones computed by CCA. In practice, however, we observe that the proposed combination gives much better cross-modality retrieval results (see Sect. 5).

Our design requires backpropagating errors through the analytical computation of the CCA projection matrices. DCCA [2] does not cover this, since projecting the data is not necessary for optimizing the TNO. In the remainder of this section, we discuss how to establish gradient flow (backpropagation) through CCA's optimal projection matrices. In particular, we require the partial derivatives $\frac{\partial \mathbf{A}^{*}}{\partial \mathbf{x}, \mathbf{y}}$ and $\frac{\partial \mathbf{B}^{*}}{\partial \mathbf{x}, \mathbf{y}}$ of the projections with respect to their input representations $\mathbf{x}$ and $\mathbf{y}$. This will allow us to use CCA as a layer within a multi-modality neural network, instead of as a final objective (TNO) for correlation maximization only.

\subsection{Gradient of CCA projections}

As mentioned above, we can compute the canonical correlation along with the optimal projection matrices from the singular value decomposition $\mathbf{T}=\Sigma_{x x}^{-1 / 2} \Sigma_{x y} \Sigma_{y y}^{-1 / 2}=$ $\mathbf{U} \operatorname{diag}(\mathbf{d}) \mathbf{V}^{\prime}$. Specifically, we obtain the correlation as $\operatorname{corr}\left(\mathbf{A}^{* \prime} \mathbf{x}, \mathbf{B}^{* \prime} \mathbf{y}\right)=\sum_{i} d_{i}$, and the projections as $\mathbf{A}^{*}=$ $\Sigma_{x x}^{-1 / 2} \mathbf{U}$ and $\mathbf{B}^{*}=\Sigma_{y y}^{-1 / 2} \mathbf{V}$. For DCCA, it suffices to compute the gradient of the total correlation wrt. $\mathbf{x}$ and $\mathbf{y}$ in order to backpropagate it through the two networks $f$ and $g$. Using the chain rule, Andrew et al. decompose this into the gradients of the total correlation wrt. $\Sigma_{x x}, \Sigma_{x y}$ and $\Sigma_{y y}$, and the gradients of those wrt. $\mathbf{x}$ and $\mathbf{y}$ [2]. Their derivations of the former make use of the fact that both the gradient of $\sum_{i} d_{i}$ wrt. $\mathbf{T}$ and the gradient of $\|\mathbf{T} \mid\|_{\text {tr }}$ (the trace norm objective in Eq. (7)) wrt. $\mathbf{T}^{\prime} \mathbf{T}$ have a simple form; see Section 7 in [2] for details.

In our case where we would like to backpropagate errors through the CCA transformations, we instead need the gradients of the projected data $\mathbf{x}^{*}=\mathbf{A}^{* \prime} \mathbf{x}$ and $\mathbf{y}^{*}=\mathbf{B}^{* \prime} \mathbf{y}$ wrt. $\mathbf{x}$ and $\mathbf{y}$, which requires the partial derivatives $\frac{\partial \mathbf{A}^{*}}{\partial \mathbf{x}, \mathbf{y}}$ and $\frac{\partial \mathbf{B}^{*}}{\partial \mathbf{x}, \mathbf{y}}$. We could again decompose this into the gradients wrt. $\mathbf{T}$, the gradients of $\mathbf{T}$ wrt. $\Sigma_{x x}, \Sigma_{x y}$ and $\Sigma_{y y}$ and the gradients of those wrt. $\mathbf{x}$ and $\mathbf{y}$. However, while the gradients of $\mathbf{U}$ and $\mathbf{V}$ wrt. $\mathbf{T}$ are known [22], they involve solving $O\left(\left(d_{x} d_{y}\right)^{2}\right)$ linear $2 \times 2$ systems. Instead, we reformulate the solution to use two symmetric eigendecompositions $\mathbf{T T}^{\prime}=\mathbf{U} \operatorname{diag}(\mathbf{e}) \mathbf{U}^{\prime}$ and $\mathbf{T}^{\prime} \mathbf{T}=\mathbf{V} \operatorname{diag}(\mathbf{e}) \mathbf{V}^{\prime}$ (Equation 270 in [24]). This gives us the same left and right eigenvectors we would obtain from the SVD, along with the squared singular values $\left(e_{i}=d_{i}^{2}\right)$. The gradients of eigenvectors of symmetric real eigensystems have a simple form [17] and both $\mathbf{T} \mathbf{T}^{\prime}$ and $\mathbf{T}^{\prime} \mathbf{T}$ are differentiable wrt. $\mathbf{x}$ and $\mathbf{y}$.

To summarize: in order to obtain an efficiently computable definition of the gradient for CCA projections, we have reformulated the forward pass (the computation of the CCA transformations). Our formulation using two eigendecompositions translates into a series of computation steps that are differentiable in a graph-based, auto-differentiating math compiler such as Theano [28], which, together with the chain rule, gives an efficient implementation of the CCA layer gradient for training our network. ${ }^{2}$ For a detailed description of

\footnotetext{
2 The code of our implementation of the CCA layer is available at https://github.com/CPJKU/cca_layer.
} 


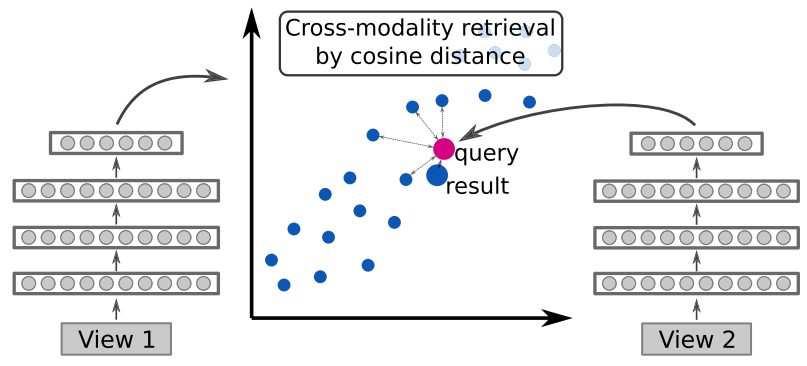

Fig. 3 Sketch of cross-modality retrieval. The blue dots are the embedded candidate samples. The red dot is the embedding of the search query. The larger blue dot highlights the closest candidate selected as the retrieval result (colour figure online)

the CCA layer forward pass, we refer to Algorithm 1 in the "Appendix" of this article. As the technical implementation is not straight-forward, we also discuss the crucial steps in the "Appendix".

Thus, we now have the means to benefit from the optimal CCA projections but still optimize for a task-specific objective. In particular, we utilize the pairwise ranking loss of Eq. (8) on top of an intermediate CCA embedding projection layer. We denote the proposed retrieval network of Fig. 1c as $C C A L-\mathcal{L}_{\text {rank }}$ in our experiments $(C C A L$ refers to CCA Layer).

\section{Experiments}

We evaluate our approach $\left(C C A L-\mathcal{L}_{\text {rank }}\right)$ in cross-modality retrieval experiments on two image-to-text and one audio-tosheet-music dataset. Additionally, we provide results on two zero-shot text-to-image retrieval scenarios proposed in [25]. For comparison, we consider the approach of [31] (DCCA2015), our own implementation of the TNO (denoted by $D C C A$ ), as well as the freely learned projection embeddings (Learned- $\mathcal{L}_{\text {rank }}$ ) optimizing the ranking loss of [15].

The task for all three datasets is to retrieve the correct counterpart when given an instance of the other modality as a search query. For retrieval, we use the cosine distance in embedding space for all approaches. First, we embed all candidate samples of the target modality into the retrieval embedding space. Then, we embed the query element $\mathbf{y}$ with the second network and select its nearest-neighbor $\mathbf{x}_{j}$ of the target modality. Fig. 3 shows a sketch of this retrieval by embedding space learning paradigm.

As evaluation measures, we consider the Recall@k(R@k in \%) as well as the Median Rank (MR) and the Mean Reciprocal Rank (MRR in \%). The R@k rate (higher is better) is the ratio of queries which have the correct corresponding counterpart in the first $k$ retrieval results. The $M R$ (lower is better) is the median position of the target in a similarity-ordered list of available candidates. Finally,
Table 1 Example images for Flickr30k (top) and IAPR TC-12 (bottom)

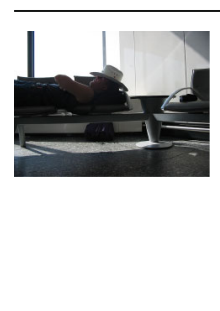

A man in a white cowboy hat reclines in front of a window in an airport

A young man rests on an airport seat with a cowboy hat over his face

A woman relaxes on a couch, with a white cowboy hat over her head

A man is sleeping inside on a bench with his hat over his eyes

A person is sleeping at an airport with a hat on their head

A green and brown embankment with brown houses on the right and a light brown sandy beach at the dark blue sea on the left; a dark mountain range behind it and white clouds in a light blue sky in the background

we define the MRR (higher is better) as the mean value of $1 /$ rank over all queries where rank is again the position of the target in the similarity-ordered list of available candidates.

\subsection{Image-text retrieval}

In the first part of our experiments, we consider Flickr30k and IAPR TC-12, two publicly available datasets for image-text cross-modality retrieval. Flickr30k consists of image-caption pairs, where each image is annotated with five different textual descriptions. The train-validation-test split for Flickr30k is 28000-1000-1000. In terms of evaluation setup, we follow Protocol 3 of [31] and concatenate the five available captions into one, meaning that only one, but richer text annotation remains per image. This is done for all three sets of the split. The second image-text dataset, IAPR TC-12, contains 20000 natural images where only one-but compared to Flickr30k more detailed - caption is available for each image. As no predefined train-validation-test split is provided, we randomly select 1000 images for validation and 2000 for testing, and keep the rest for training. [31] also use 2000 images for testing, but did not explicitly mention holdout images for validation. Table 1 shows an example image along with its corresponding captions or caption for either dataset.

The input to our networks is a 4096-dimensional image feature vector along with a corresponding text vector representation which has dimensionality 5793 for Flickr30k and 2048 for IAPR TC-12. The image embedding is computed from the last hidden layer of a network pretrained on ImageNet [7] (layer $f c 7$ of $C N N \_S$ by [4]). In terms of text pre-processing, we follow [31], tokenizing and lemmatizing the raw captions as the first step. Based on the lemmatized captions, we compute $l 2$-normalized TF/IDF-vectors, omit- 
Table 2 Retrieval results on IAPR TC-12. "DCCA-2015" is taken from [31]

Table 3 Retrieval results on Flickr30k. "DCCA-2015" is taken from [31]

\begin{tabular}{|c|c|c|c|c|c|c|c|c|c|c|}
\hline \multirow[t]{2}{*}{ Method } & \multicolumn{5}{|c|}{ Image-to-text } & \multicolumn{5}{|c|}{ Text-to-image } \\
\hline & $\mathrm{R} @ 1$ & $\mathrm{R} @ 5$ & $\mathrm{R} @ 10$ & MR & MRR & $\mathrm{R} @ 1$ & $\mathrm{R} @ 5$ & R@10 & MR & MRR \\
\hline DCCA-2015 & 30.2 & 57.0 & - & - & 42.6 & 29.5 & 60.0 & - & - & 41.5 \\
\hline DCCA & 31.0 & 58.7 & 70.4 & 3.6 & 43.9 & 29.5 & 58.2 & 70.5 & 4.0 & 42.7 \\
\hline Learned- $\mathcal{L}_{\text {rank }}$ & 22.3 & 50.7 & 63.8 & 5.2 & 35.7 & 21.6 & 50.1 & 63.3 & 5.5 & 35.1 \\
\hline CCAL- $\mathcal{L}_{\text {rank }}$ & 31.6 & 61.0 & 72.2 & 3.0 & 45.0 & 29.6 & 60.0 & 72.2 & 3.6 & 43.5 \\
\hline
\end{tabular}

\begin{tabular}{|c|c|c|c|c|c|c|c|c|c|c|}
\hline \multirow[t]{2}{*}{ Method } & \multicolumn{5}{|c|}{ Image-to-text } & \multicolumn{5}{|c|}{ Text-to-image } \\
\hline & $\mathrm{R} @ 1$ & $\mathrm{R} @ 5$ & R@ 10 & MR & MRR & $\mathrm{R} @ 1$ & $\mathrm{R} @ 5$ & R@10 & MR & MRR \\
\hline DCCA-2015 & 27.9 & 56.9 & 68.2 & 4 & - & 26.8 & 52.9 & 66.9 & 4 & - \\
\hline DCCA & 31.6 & 59.2 & 69.3 & 3.3 & 44.2 & 30.3 & 58.3 & 69.2 & 3.8 & 43.1 \\
\hline Learned- $\mathcal{L}_{\text {rank }}$ & 23.7 & 50.5 & 63.0 & 5.3 & 36.3 & 23.6 & 51.0 & 62.5 & 5.2 & 36.5 \\
\hline CCAL- $\mathcal{L}_{\text {rank }}$ & 32.0 & 59.2 & 70.4 & 3.2 & 44.8 & 29.9 & 58.8 & 70.2 & 3.7 & 43.3 \\
\hline
\end{tabular}

ting words with an overall occurrence smaller than five for Flickr30k and three for IAPR TC-12, respectively. The image representation is processed by a linear dense layer with 128 units, which will also be the dimensionality $k$ of the resulting retrieval embedding. The text vector is fed through two batch-normalized [11] dense layers of 1024 units each and the ELU activation function [6]. As a last layer for the text representation network, we again apply a dense layer with 128 linear units.

For a fair comparison, we keep the structure and number of parameters of all networks in our experiments the same. The only difference between the networks are the objectives and the hyper-parameters used for optimization. Optimization is performed using Stochastic Gradient Descent (SGD) with the adam update rule [14] (for details please see our "Appendix").

Table 2 lists our results on IAPR TC-12. Along with our experiments, we also show the results reported in [31] as a reference (DCCA-2015). However, a direct comparison to our results may not be fair: DCCA-2015 uses a different ImageNet-pretrained network for the image representation, and finetunes this network while we keep it fixed. This is because our interest is in comparing the methods in a stable setting, not in obtaining the best possible results. Our implementation of the TNO (DCCA) uses the same objective as $D C C A-2015$, but is trained using the same network architecture as our remaining models and permits a direct comparison. Additionally, we repeat each of the experiments 10 times with different initializations and report the mean for each of the evaluation measures.

When taking a closer look at Table 2, we observe that our results achieved by optimizing the TNO (DCCA) surpass the results reported in [31]. We already discussed above that the two versions are not directly comparable. However, given this result, we consider our implementation of DCCA as a valid baseline for our experiments in Sect.5.2 where no results are available in the literature. When looking at the performance of $C C A L-\mathcal{L}_{\text {rank }}$ we further observe that it outperforms all other methods, although the difference to DCCA is not pronounced for all of the measures. Comparing $C C A L-\mathcal{L}_{\text {rank }}$ with the freely learned projection matrices (Learned- $\mathcal{L}_{\text {rank }}$ ) we observe a much larger performance gap. This is interesting, as in principle the learned projections could converge to exactly the same solution as $C C A L-\mathcal{L}_{\text {rank }}$. We take this as a quantitative confirmation that the learning process benefits from CCA's optimal projection matrices.

In Table 3, we list our results on the Flickr30k dataset. As above, we show the retrieval performances of [31] as a baseline along with our results and observe similar behavior as on IAPR TC-12. Again, we point out the poor performance of the freely learned projections (Learned- $\mathcal{L}_{\text {rank }}$ ) in this experiment. Keeping this observation in mind, we will notice a different behavior in the experiments in Sect. 5.2.

Note that there are various other methods reporting results on Flickr30k [13,15,18,27] which partly surpass ours, for example by using more elaborate processing of the textual descriptions or more powerful ImageNet models. We omit these results as we focus on the comparison of DCCA and freely learned projections with the proposed CCA projection embedding layer.

\subsection{Audio-sheet-music retrieval}

For the second set of experiments, we consider the Nottingham piano midi dataset [3]. The dataset is a collection of midi files split into train, validation and test set already used by [8] for experiments on end-to-end score-following in sheet-music images. Here, we tackle the problem of audiosheet-music retrieval, i.e., matching short snippets of music (audio) to corresponding parts in the sheet music (image). Figure 4 shows examples of such correspondences. 


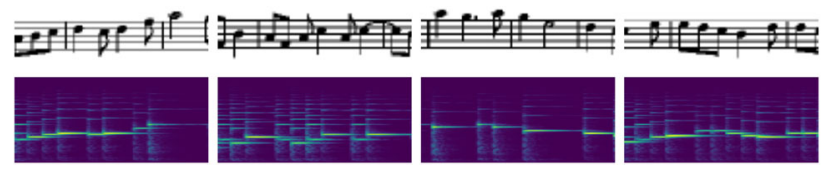

Fig. 4 Example of the data considered for audio-sheet-music (image) retrieval. Top: short snippets of sheet-music images. Bottom: Spectrogram excerpts of the corresponding music audio

We conduct this experiment for two reasons: First, to show the advantage of the proposed method over different domains. Second, the data and application is of high practical relevance in the domain of Music Information Retrieval (MIR). A system capable of linking sheet music (images) and the corresponding music (audio) would be useful in many content-based musical retrieval scenarios.

In terms of audio preparation, we compute log frequency spectrograms with a sample rate of $22.05 \mathrm{kHz}$, a FFT window size of 2048, and a computation rate of 31.25 frames per second. These spectrograms (136 frequency bins) are then directly fed into the audio part of the cross-modality networks. Figure 4 shows a set of audio-to-sheet correspondences presented to our network for training. One audio excerpt comprises 100 frames and the dimension of the sheet image snippet is $40 \times 100$ pixels. Overall this results in 270,705 train, 18,046 validation and 16,042 test audio-sheetmusic pairs. This is an order of magnitude more training data than for the image-to-text datasets of the previous section.

In the experiments in Sect.5.1, we relied on pretrained ImageNet features and relatively shallow fully connected text-feature processing networks. The model here differs from this, as it consists of two deep convolutional networks learned entirely from scratch. Our architecture is a VGG-style [26] network consisting of sequences of $3 \times 3$ convolution stacks followed by $2 \times 2$ max pooling. To reduce the dimensionality to the desired correlation space dimensionality $k$ (in this case 32 ), we insert as a final building block a $1 \times 1$ convolution having $k$ feature maps followed by global average pooling [16] (for further architectural details we again refer to the appendix of this manuscript).

Table 4 lists our result on audio-to-sheet-music retrieval. As in the experiments on images and text, the proposed CCA projection embedding layer trained with pairwise ranking loss outperforms the other models. Recalling the results from Sect.5.1, we observe an increased performance of the freely learned embedding projections. On measures such as R@5 or R@10 it achieves similar to or better performance than $D C C A$. One of the reasons for this could be the fact that there is an order of magnitude more training data available for this task to learn the projection embedding from random initialization. Still, our proposed combination of both concepts $\left(C C A L-\mathcal{L}_{\text {rank }}\right)$ achieves highest retrieval scores.

\subsection{Performance in small data regime}

The above results suggest that the benefit of using a CCA projection layer $\left(C C A L-\mathcal{L}_{\text {rank }}\right)$ over a freely learned projection becomes most evident when few training data is available. To examine this assumption, we repeat the audio-to-sheetmusic experiment of the previous section, but use only $10 \%$ of the original training data $(\approx 27000$ samples $)$. We stress the fact that the learned embedding projection of Learned$\mathcal{L}_{\text {rank }}$ could converge to exactly the same solution as the CCA projections of $C C A L-\mathcal{L}_{\text {rank }}$. Table 5 summarizes the low data regime results for the three methods. Consistent with our hypothesis, we observe a larger gap between Learned$\mathcal{L}_{\text {rank }}$ and $C C A L-\mathcal{L}_{\text {rank }}$ compared to the one obtained with all training data in Table 4 . We conclude that a network might be able to learn suitable embedding projections when sufficient training data is available. However, when having fewer training samples, the proposed CCA projection layer strongly supports embedding space learning. In addition, we also looked into the retrieval performance of Learned $-\mathcal{L}_{\text {rank }}$ and $C C A L-\mathcal{L}_{\text {rank }}$ on the training set and observe comparable performance. This indicates that the CCA layer also acts as a regularizer and helps to generalize to unseen samples.

\subsection{Zero-shot image-text retrieval}

Our last set of experiments focuses on a slightly modified retrieval setting, namely image-text zero-shot retrieval [25]. Given a set of image-text pairs originating from $C$ different categories the data is split into a class-disjoint training, validation and test sets having no categorical overlap. This implies that at test time we aim to retrieve images from textual queries describing categories (semantic concepts) never seen before, neither for training, nor for validation.

Reed et al. [25] collected and provided textual descriptions for two publicly available datasets, the CUB-200 bird image
Table 4 Retrieval results on Nottingham dataset (audio-to-sheet-music retrieval)

\begin{tabular}{|c|c|c|c|c|c|c|c|c|c|c|}
\hline \multirow[t]{2}{*}{ Method } & \multicolumn{5}{|c|}{$\underline{\text { Sheet-to-audio }}$} & \multicolumn{5}{|c|}{ Audio-to-sheet } \\
\hline & $\mathrm{R} @ 1$ & $\mathrm{R} @ 5$ & R@ 10 & MR & MRR & $\mathrm{R} @ 1$ & R@5 & R@10 & MR & MRR \\
\hline DCCA & 42.0 & 88.2 & 93.3 & 2 & 62.2 & 44.6 & 87.9 & 93.2 & 2 & 63.5 \\
\hline Learned- $\mathcal{L}_{\text {rank }}$ & 40.7 & 89.6 & 95.6 & 2 & 61.7 & 41.4 & 88.9 & 95.4 & 2 & 61.9 \\
\hline CCAL- $\mathcal{L}_{\text {rank }}$ & 44.1 & 93.3 & 97.7 & 2 & 65.3 & 44.5 & 91.6 & 96.7 & 2 & 64.9 \\
\hline
\end{tabular}


Table 5 Retrieval results on audio-to-sheet-music retrieval when using only $10 \%$ of the train data

\begin{tabular}{|c|c|c|c|c|c|c|c|c|c|c|}
\hline \multirow[t]{2}{*}{ Method } & \multicolumn{5}{|c|}{$\underline{\text { Sheet-to-audio }}$} & \multicolumn{5}{|c|}{ Audio-to-sheet } \\
\hline & $\mathrm{R} @ 1$ & $\mathrm{R} @ 5$ & R@10 & MR & MRR & $\mathrm{R} @ 1$ & $\mathrm{R} @ 5$ & $\mathrm{R} @ 10$ & MR & MRR \\
\hline DCCA & 20.0 & 53.6 & 65.4 & 5 & 35.3 & 22.7 & 54.7 & 65.8 & 4 & 37.3 \\
\hline Learned- $\mathcal{L}_{\text {rank }}$ & 11.3 & 35.2 & 47.6 & 12 & 23.0 & 12.6 & 35.2 & 47.2 & 12 & 23.7 \\
\hline CCAL- $\mathcal{L}_{\text {rank }}$ & 22.2 & 59.2 & 70.7 & 4 & 38.8 & 25.0 & 59.3 & 70.9 & 4 & 40.4 \\
\hline
\end{tabular}

Table 6 Zero-shot retrieval results on cub and flowers

\begin{tabular}{lll}
\hline Method & Flowers & Birds \\
\hline Attributes [25] & - & 50.0 \\
Word2Vec [25] & 52.1 & 33.5 \\
Word CNN [25] & 56.3 & 43.3 \\
Word CNN-RNN [25] & 59.6 & 48.7 \\
Word CNN + CCAL & 62.2 & 52.2 \\
Word CNN-RNN + CCAL & 64.0 & 49.8 \\
\hline
\end{tabular}

For the Birds dataset, as an alternative to the textual descriptions, there are manually created fine-grained attributes available for each of the images. When relying on these attributes Reed et al. report state-of-the-art results on the dataset [25] not reached by their text processing neural networks.

In the bottom part of Table 6, we report the performance of the same architectures optimized using our proposed CCA layer in combination with a pairwise ranking loss. We observe that the CCA layer is able to improve the performance of both models on both datasets. The gain in retrieval performance within a model class is largest for the convolution only $(\mathrm{CNN})$ text processing models $(\approx 9 \%$ points for the Flowers dataset and $\approx 6$ for CUB). For the birds dataset the Word $C N N+C C A L$ even outperforms the models relying on manually encoded attributes by achieving an AP@50 of 52.2 .

\section{Discussion and conclusion}

We have shown how to use the optimal projection matrices of CCA as the weights of an embedding layer within a multi-view neural network. With this CCA layer, it becomes possible to optimize for a specialized loss function (e.g., related to a retrieval task) on top of this, exploiting the correlation properties of a latent space provided by CCA. As this requires to establish gradient flow through CCA, we formulate it to allow easy computation of the partial derivatives $\frac{\partial \mathbf{A}^{*}}{\partial \mathbf{x}, \mathbf{y}}$ and $\frac{\partial \mathbf{B}^{*}}{\partial \mathbf{x}, \mathbf{y}}$ of CCA's projection matrices $\mathbf{A}^{*}$ and $\mathbf{B}^{*}$ with respect to the input data $\mathbf{x}$ and $\mathbf{y}$. With this formulation, we can incorporate CCA as a building block within multi-modality neural networks that produces maximally correlated projections of its inputs. In our experiments, we use this building 
block within a cross-modality retrieval setting, optimizing a network to minimize a cosine distance-based pairwise ranking loss of the componentwise-correlated CCA projections. Experimental results show that when using the cosine distance for retrieval (as is common for correlated views), this is superior to optimizing a network for maximally correlated projections (as done in DCCA), or not using CCA at all. This observation holds in our experiments on a variety of different modality pairs as well as two different retrieval scenarios.

When investigating the experimental results in more detail, we find that the correlation-based methods (DCCA, CCAL) consistently outperform the models that learn the embedding projections from scratch. A direct comparison of DCCA with the proposed CCAL- $\mathcal{L}_{\text {rank }}$ reveals two learning scenarios where CCAL- $\mathcal{L}_{\text {rank }}$ is superior: (1) the low data regime, where we found that the CCA layer acts as a strong regularizer to prevent over-fitting; (2) when learning the entire retrieval representation (network parameterization) from scratch, not relying on pretrained or hand-crafted features (see Sect. 5.2). Our intuition on this is that incorporating the task-specific retrieval objective already during training encourages the networks to learn embedding representations that are beneficial for retrieval at test time. This is the important conceptual difference compared to the Trace Norm Objective (TNO) of DCCA, which does not focus on the retrieval task. However, when using the CCA layer we also inherit one drawback of the pairwise ranking loss, which is the additional hyper-parameter (margin $\alpha$ ) that needs to be determined on the validation set.

Finally, we would like to emphasize that our CCA layer is a general network component which could provide a useful basis for further research, e.g., as an intermediate processing step for learning binary cross-modality retrieval representations.

Acknowledgements Open access funding provided by Johannes Kepler University Linz.

Open Access This article is distributed under the terms of the Creative Commons Attribution 4.0 International License (http://creativecomm ons.org/licenses/by/4.0/), which permits unrestricted use, distribution, and reproduction in any medium, provided you give appropriate credit to the original author(s) and the source, provide a link to the Creative Commons license, and indicate if changes were made.

\section{Appendix}

\section{Implementation details}

Backpropagating the errors through the CCA projection matrices is not trivial. The optimal CCA projection matrices are given by $\mathbf{A}^{*}=\Sigma_{x x}^{-1 / 2} \mathbf{U}$ and $\mathbf{B}^{*}=\Sigma_{y y}^{-1 / 2} \mathbf{V}$, where $\mathbf{U}$ and $\mathbf{V}$ are derived from the singular value decomposition of $\mathbf{T}=\Sigma_{x x}^{-1 / 2} \Sigma_{x y} \Sigma_{y y}^{-1 / 2}=\mathbf{U} \operatorname{diag}(\mathbf{d}) \mathbf{V}^{\prime}$ (see Sect. 2). The proposed model needs to backpropagate the errors through the CCA transformations, i.e., it requires the gradients of the projected data $\mathbf{x}^{*}=\mathbf{A}^{* \prime} \mathbf{x}$ and $\mathbf{y}^{*}=\mathbf{B}^{* \prime} \mathbf{y}$ wrt. $\mathbf{x}$ and $\mathbf{y}$. Applying the chain rule, this further requires the gradients of $\mathbf{U}$ and $\mathbf{V}$ wrt. T, and the gradients of $\mathbf{T}, \Sigma_{x x}^{-1 / 2}, \Sigma_{x y}$ and $\Sigma_{y y}^{-1 / 2}$ wrt. $\mathbf{x}$ and $\mathbf{y}$.

The main technical challenge is that common autodifferentiation tools such as Theano [28] or Tensor Flow [1] do not provide derivatives for the inverse squared root and singular value decomposition of a matrix. ${ }^{3}$ To overcome this, we replace the inverse squared root of a matrix by using its Cholesky decomposition as described in [9]. Furthermore, we note that the singular value decomposition is required to obtain the matrices $\mathbf{U}$ and $\mathbf{V}$, but in fact those matrices can alternatively be obtained by solving the eigendecomposition of $\mathbf{T} \mathbf{T}^{\prime}=\mathbf{U} \operatorname{diag}(\mathbf{e}) \mathbf{U}^{\prime}$ and $\mathbf{T}^{\prime} \mathbf{T}=\mathbf{V} \operatorname{diag}(\mathbf{e}) \mathbf{V}^{\prime}[24$, Eq. 270]. This yields the same left and right eigenvectors we would obtain from the SVD (except for possibly flipped signs, which are easy to fix), along with the squared singular values $\left(e_{i}=d_{i}^{2}\right)$. Note that $\mathbf{T} \mathbf{T}^{\prime}$ and $\mathbf{T}^{\prime} \mathbf{T}$ are symmetric, and that the gradients of eigenvectors of symmetric real eigensystems have a simple form [17, Eq. 7]. Furthermore, TT $^{\prime}$ and $\mathbf{T}^{\prime} \mathbf{T}$ are differentiable wrt. $\mathbf{x}$ and $\mathbf{y}$, enabling a sufficiently efficient implementation in a graph-based, auto-differentiating math compiler. ${ }^{4}$

The following section provides a detailed description of the implementation of the CCA layer.

\section{Forward pass of CCA projection layer}

For easier reproducibility, we provide a detailed description of the forward pass of the proposed CCA layer in Algorithm 1. To train the model, we need to propagate the gradient through the CCA layer (backward pass). We rely on auto-differentiation tools (in particular, Theano) implementing the gradient for each individual computation step in the forward pass, and connecting them using the chain rule.

The layer itself takes the latent feature representations (a batch of $m$ paired vectors $\mathbf{X} \in \mathbb{R}^{d_{x} \times m}$ and $\mathbf{Y} \in \mathbb{R}^{d_{y} \times m}$ ) of the two network pathways $f$ and $g$ as input and projects them with CCA's analytical projection matrices. At train time, the layer uses the optimal projections computed from the current batch. When applying the layer at test time, it uses the statistics and projections remembered from last training batch (which can of course be recomputed on a larger training batch to get more stable estimate).

\footnotetext{
${ }^{3}$ Note that this is not relevant for the DCCA model introduced in [2] because it only derives the CCA projections after optimizing the TNO.

4 The code of our implementation of the CCA layer is available at https://github.com/CPJKU/cca_layer.
} 


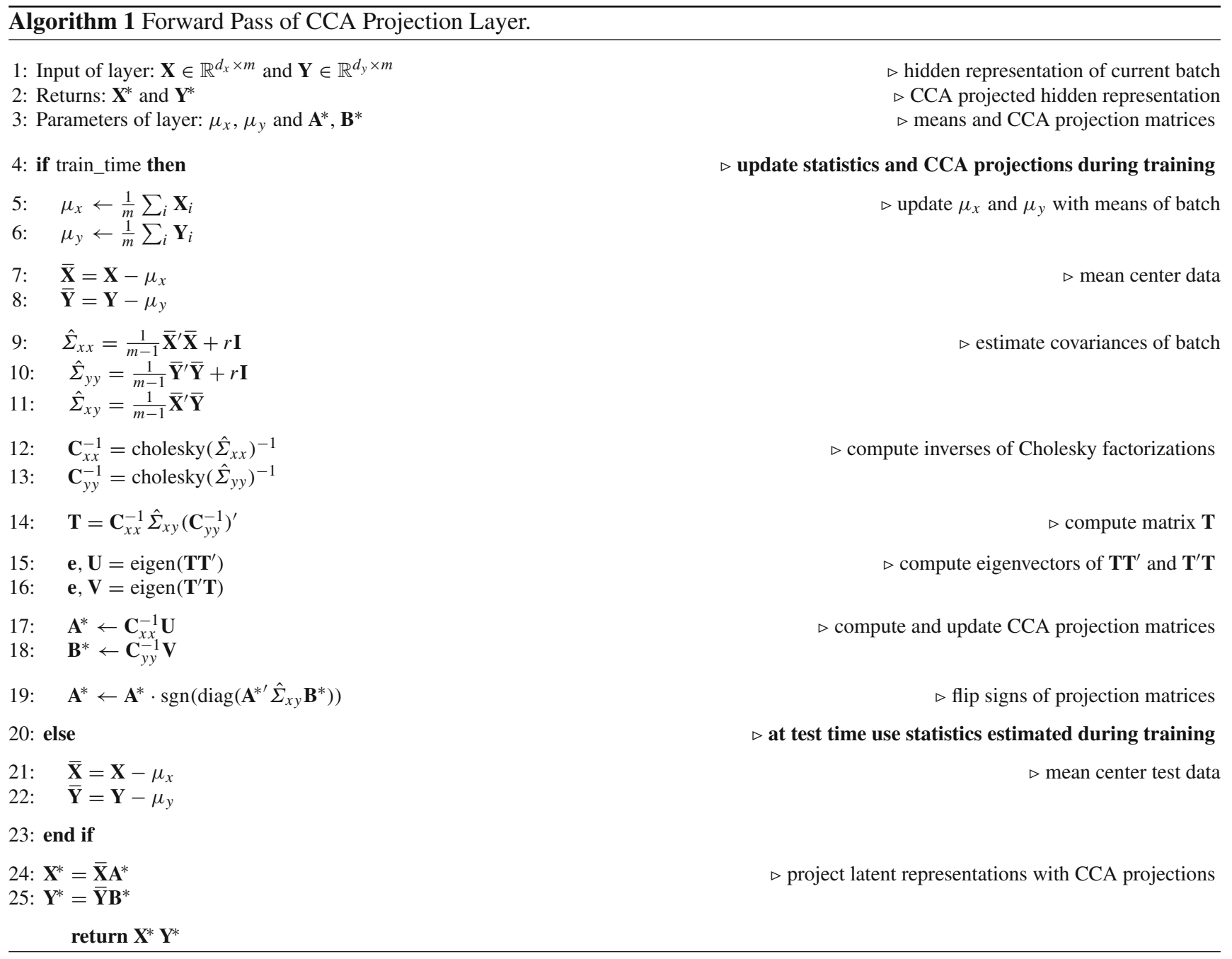

As not all of the computation steps are obvious, we provide further details for the crucial ones. In line 12 and 13, we compute the Cholesky factorization instead of the matrix square root, as the latter has no gradients implemented in Theano. As a consequence, we need to transpose $\mathbf{C}_{y y}^{-1}$ when computing $\mathbf{T}$ in line 14 [9]. In line 15 and 16, we compute two eigendecompositions instead of one singular value decomposition (which also has no gradients implemented in Theano). In line 19, we flip the signs of first projection matrix to match the second to only have positive correlations. This property is required for retrieval with cosine distance. Finally, in line 24 and 25 , the two views get projected using $\mathbf{A}^{*}$ and $\mathbf{B}^{*}$. At test time, we apply the projections computed and stored during training (line 17).

\section{Investigations on correlation structure}

As an additional experiment we investigate the correlation structure of the learned representations for all three paradigms. For that purpose we compute the topmost hid- den representation $\mathbf{x}$ and $\mathbf{y}$ of the audio-sheet-music-pairs and estimate the canonical correlation coefficients $d_{i}$ of the respective embedding spaces. For the present example, this yields 32 coefficients which is the dimensionality $k$ of our retrieval embedding space. Figure 6 compares the correlation coefficients where 1.0 is the maximum value reachable. The most prominent observation in Fig. 6 is the high correlation coefficients of the representation learned with $D C C A$. This structure is expected as the TNO focuses solely on correlation maximization. However, when recalling the results of Table 4 we see that this does not necessarily lead to the best retrieval performance. The freely learned embedding Learned- $\mathcal{L}_{\text {rank }}$ shows overall the lowest correlation but achieves comparable results to DCCA on this dataset. In terms of overall correlation, CCAL- $\mathcal{L}_{\text {rank }}$ is situated inbetween the two other approaches. We have seen in all our experiments that combining both concepts in a unified retrieval paradigm yields best retrieval performance over different application domains as well as data regimes. We take 


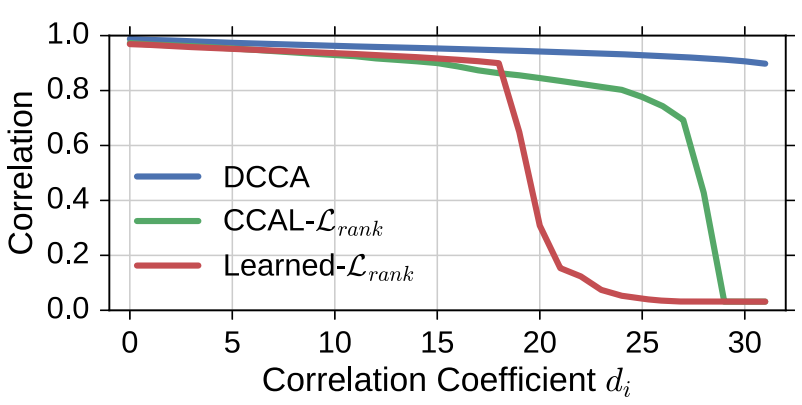

Fig. 6 Comparison of the 32 correlation coefficients $d_{i}$ (the dimensionality of the retrieval space is 32) of the topmost hidden representations $\mathbf{x}$ and $\mathbf{y}$ of the audio-to-sheet-music dataset and the respective optimization paradigm. The maximum correlation possible is 1.0 for each coefficient

this as evidence that componentwise-correlated projections support cosine distance-based embedding space learning.

\section{Architecture and optimization}

In the following, we proved additional details for our experiments carried out in Sect. 5 .

\section{Image-text retrieval}

We start training with an initial learning rate of either 0.001 (all models on IAPR TC-12 and Flickr30k Learned- $\mathcal{L}_{\text {rank }}$ ) or 0.002 (Flickr30k DCCA and CCAL- $\left.\mathcal{L}_{\text {rank }}\right)^{5}$. In addition, we apply $0.0001 L 2$ weight decay and set the batch size to 1000 for all models. The parameter $\alpha$ of the ranking loss in Eq. (8) is set to 0.5 . After no improvement on the validation set for 50 epochs, we divide the learning rate by 10 and reduce the patience to 10 . This learning rate reduction is repeated three times.

\section{Audio-sheet-music retrieval}

Table 7 provides details on our audio-sheet-music retrieval architecture.

As in the experiments on images and text, we optimize our networks using adam with an initial learning rate of 0.001 and batch size 1000. The refinement strategy is the same, but no weight decay is applied and the margin parameter $\alpha$ of the ranking loss is set to 0.7 .

\section{Zero-shot retrieval}

Tables 8 and 9 provide details on the architectures used for our zero-shot retrieval experiments carried out in Sect. 5.4. The general architectures follow Reed et al. [25] but are optimized

\footnotetext{
$\overline{5}$ The initial learning rate and parameter $\alpha$ are determined by grid search on the evaluation measure MRR on the validation set.
}

Table 7 Architecture of audio-sheet-music model

\begin{tabular}{ll}
\hline Sheet-image $40 \times 100$ & Spectrogram $136 \times 100$ \\
\hline $2 \times$ Conv(3, pad-1)-16 & $2 \times$ Conv(3, pad-1)-16 \\
BN-ELU + MP(2) & BN-ELU + MP(2) \\
$2 \times$ Conv(3, pad-1)-32 & $2 \times$ Conv(3, pad-1)-32 \\
BN-ELU + MP(2) & BN-ELU + MP(2) \\
$2 \times$ Conv(3, pad-1)-64 & $2 \times$ Conv(3, pad-1)-64 \\
BN-ELU + MP(2) & BN-ELU + MP(2) \\
$2 \times$ Conv(3, pad-1)-64 & $2 \times$ Conv(3, pad-1)-64 \\
BN-ELU + MP(2) & BN-ELU + MP(2) \\
Conv(1, pad-0)-32-BN & Conv(1, pad-0)-32-BN \\
Global average pooling & Global average pooling \\
\hline
\end{tabular}

Respective optimization target

$B N$ batch normalization, $E L U$ exponential linear unit, $M P$ max pooling, conv(3, pad-1)-16: $3 \times 3$ convolution, 16 feature maps and padding 1

Table 8 Architecture of zero-shot retrieval CNN

\begin{tabular}{ll}
\hline ImagenNet feature 1024 & Text $V S \times 30 \times 1$ \\
\hline FC(1024)-BN-ELU & $1 \times \operatorname{Conv}(3$, pad-same)-256 \\
FC(1024)-BN-ELU & BN-ELU + MP(3,1) \\
FC(64) & $2 \times \operatorname{Conv}(3$, pad-valid)-256 \\
& FC(1024)-BN-ELU \\
& FC(64) \\
\hline
\end{tabular}

Respective optimization target

$V S$ vocabulary size, $B N$ batch normalization, $E L U$ exponential linear unit, $M P$ max pooling, $\operatorname{Conv}(3$, pad-1)-16: $3 \times 3$ convolution, 16 feature maps and padding 1

Table 9 Architecture of zero-shot retrieval CRNN

\begin{tabular}{|c|c|}
\hline ImagenNet feature 1024 & Text $V S \times 30 \times 1$ \\
\hline FC(1024)-BN-ELU & $1 \times \operatorname{Conv}(3$, pad-same $)-256$ \\
\hline FC(1024)-BN-ELU & $\mathrm{BN}-\mathrm{ELU}+\mathrm{MP}(3,1)$ \\
\hline \multirow[t]{4}{*}{$\mathrm{FC}(64)$} & $2 \times \operatorname{Conv}(3$, pad-valid $)-256$ \\
\hline & GRU-RNN(512) \\
\hline & Temporal average pooling \\
\hline & $\mathrm{FC}(64)$ \\
\hline
\end{tabular}

Respective optimization target

with a pairwise ranking loss in combination with our proposed CCA layer. The dimensionality of the retrieval space is fixed to 64 and both models are again optimized with adam and a batch size of 1000 . The learning rate is set to 0.0007 for the CNN and 0.01 for the CRNN and. The margin parameter $\alpha$ of the ranking loss is set to 0.2 . In addition, we apply a weight decay of 0.0001 on all trainable parameters of the network for regularization. 


\section{References}

1. Abadi M, Agarwal A, Barham P, Brevdo E, Chen Z, Citro C, Corrado GS, Davis A, Dean J, Devin M et al (2016) Tensorflow: large-scale machine learning on heterogeneous distributed systems. arXiv preprint arXiv: 1603.04467

2. Andrew G, Arora R, Bilmes J, Livescu K (2013) Deep canonical correlation analysis. In: Proceedings of the international conference on machine learning, pp 1247-1255

3. Boulanger-Lewandowski N, Bengio Y, Vincent P (2012) Modeling temporal dependencies in high-dimensional sequences: application to polyphonic music generation and transcription. In: Proceedings of the 29th international conference on machine learning (ICML12), pp 1159-1166

4. Chatfield K, Simonyan K, Vedaldi A, Zisserman A (2014) Return of the devil in the details: delving deep into convolutional nets. In: British machine vision conference

5. Chung J, Gülçehre Ç, Cho K, Bengio Y (2014) Empirical evaluation of gated recurrent neural networks on sequence modeling. CoRR, abs/1412.3555

6. Clevert D, Unterthiner T, Hochreiter S (2015) Fast and accurate deep network learning by exponential linear units (elus). In: International conference on learning representations (ICLR). arXiv: 1511.07289

7. Deng J, Dong W, Socher R, Li L-J, Li K, Fei-Fei L (2009) ImageNet: a large-scale hierarchical image database. In: CVPR09

8. Dorfer M, Arzt A, Widmer G (2016) Towards score following in sheet music images. In: Proceedings of the international society for music information retrieval conference (ISMIR)

9. Hardoon DR, Szedmak S, Shawe-Taylor J (2004) Canonical correlation analysis: an overview with application to learning methods. Neural Comput 16(12):2639-2664

10. Hermann KM, Blunsom P (2013) Multilingual distributed representations without word alignment. arXiv preprint arXiv: 1312.6173

11. Ioffe S, Szegedy C (2015) Batch normalization: accelerating deep network training by reducing internal covariate shift. CoRR, $\mathrm{abs} / 1502.03167$

12. Karpathy A, Fei-Fei L (2015) Deep visual-semantic alignments for generating image descriptions. In: Proceedings of the IEEE conference on computer vision and pattern recognition, pp 31283137

13. Karpathy A, Joulin A, Li FFF (2014) Deep fragment embeddings for bidirectional image sentence mapping. In: Advances in neural information processing systems, pp 1889-1897

14. Kingma D, Ba J (2014) Adam: a method for stochastic optimization. arXiv preprint arXiv: 1412.6980

15. Kiros R, Salakhutdinov R, Zemel RS (2014) Unifying visualsemantic embeddings with multimodal neural language models. arXiv preprint arXiv: 1411.2539

16. Lin M, Chen Q, Yan S (2013) Network in network. CoRR, abs/1312.4400

17. Magnus JR (1985) On differentiating eigenvalues and eigenvectors. Econom Theory 1(2):179-191
18. Mao J, Xu W, Yang Y, Wang J, Yuille AL (2014) Explain images with multimodal recurrent neural networks. arXiv preprint arXiv: 1410.1090

19. Mardia KV, Kent JT, Bibby JM (1979) Multivariate analysis. Probability and mathematical statistics. Academic Press, London

20. Mikolov T, Sutskever I, Chen K, Corrado GS, Dean J (2013) Distributed representations of words and phrases and their compositionality. In: Advances in neural information processing systems, pp 3111-3119

21. Nilsback M-E, Zisserman A (2008) Automated flower classification over a large number of classes. In: Proceedings of the Indian conference on computer vision, graphics and image processing

22. Papadopoulo T, Lourakis MIA (2000) Estimating the Jacobian of the singular value decomposition: theory and applications. In: Proceedings of the 6th European conference on computer vision (ECCV)

23. Pereira JC, Coviello E, Doyle G, Rasiwasia N, Lanckriet GRG, Levy R, Vasconcelos N (2014) On the role of correlation and abstraction in cross-modal multimedia retrieval. IEEE Trans Pattern Anal Mach Intell 36(3):521-535

24. Petersen KB, Pedersen MS (2012) The matrix cookbook, nov 2012. Version 20121115

25. Reed S, Akata Z, Schiele B, Lee H (2016) Deep visual-semantic alignments for generating image descriptions. In: Proceedings of the IEEE conference on computer vision and pattern recognition

26. Simonyan K, Zisserman A (2014) Very deep convolutional networks for large-scale image recognition. arXiv preprint arXiv: 1409.1556

27. Socher R, Karpathy A, Le QV, Manning CD, Ng. AY (2014) Grounded compositional semantics for finding and describing images with sentences. Trans Assoc Comput Linguist 2:207-218

28. Theano Development Team (2016) Theano: a Python framework for fast computation of mathematical expressions. arXiv e-prints, abs/1605.02688, May 2016

29. Vendrov I, Kiros R, Fidler S, Urtasun R (2016) Order-embeddings of images and language. CoRR, abs/1511.06361

30. Welinder P, Branson S, Mita T, Wah C, Schroff F, Belongie S, Perona P (2010) Caltech-UCSD Birds 200. Technical report CNSTR-2010-001, California Institute of Technology

31. Yan F, Mikolajczyk K (2015) Deep correlation for matching images and text. In: Proceedings of the IEEE conference on computer vision and pattern recognition, pp 3441-3450 